\title{
The moving learner: Object manipulation in VR improves vocabulary learning
}

Orly Fuhrman ${ }^{1,2}$, Anabel Eckerling ${ }^{1}$, Naama Friedmann ${ }^{1,3}$, Ricardo Tarrasch ${ }^{1,4 *}$, and Gal Raz ${ }^{1,5,6 *}$

1. Sagol School of Neuroscience, Tel Aviv University, Tel-Aviv, Israel

2. The Center for Educational Technology, Tel-Aviv, Israel

3. Language and Brain Lab, The Jaime and Joan Constantiner School of Education, Tel-Aviv University, Tel-Aviv, Israel

4. Department of School Counseling and Special Education, The Jaime and Joan Constantiner School of Education, Tel-Aviv University, Tel-Aviv, Israel.

5. Steve Tisch School of Film and Television, Tel Aviv University, Tel-Aviv, Israel

6. Sagol Brain Institute, Wohl Institute for Advanced Imaging, Tel-Aviv Sourasky Medical Centre, Tel Aviv, Israel

* These authors contributed equally to the work.

\section{Terms: Manipulation movement vs. irrelevant movement, virtual reality, second language} acquisition, embodied cognition

\begin{abstract}
A challenging part of foreign language acquisition is learning new vocabulary. Research has demonstrated the benefits of motor action to vocabulary learning, though these findings are rarely translated into teaching and learning practices. In this work we present a novel paradigm for vocabulary learning in a virtual reality environment, capitalizing on the prominent advantages of this technology as an 'embodied' learning tool. Hebrew-speaking adults explored a virtual room and learned the Finnish names of 40 daily objects. Every participant learned the new object names under 3 conditions: (1) Watch-only: watching the object without performing any movement (2) performing an irrelevant movement, or (3) performing a manipulation movement that implied its habitual use. Participants completed a word-picture matching test after each training block, as well as additional word-picture matching and recall tests a week after the initial training session. The overall results demonstrate improved comprehension rates for items that were learned in the manipulation movement condition compared to the irrelevant movement condition, both in the initial learning session and one week following it. These initial findings suggest that VR technology may indeed hold the potential to offer a more authentic, multisensory and motor context to efficient foreign language learning.
\end{abstract}




\section{Introduction}

What role does the body play in language learning? This question dates back to the days of Aristotle, where philosophers and scientists have wondered about the possible interaction between body and mind. Symbolic theories, which dominated the field for centuries, view thought as the manipulation of symbols and operations, far removed from bodily processes. In contrast, contenders of embodied cognition claim that cognitive processes, including language, are grounded in human experience and influence the way we construct concepts, make inferences and use language (Barsalou, 2008).

The last two decades have seen a surge in embodied cognition research, pointing to the close relationship between the sensory and motor systems and cognitive processes (for review see Barsalou, 2008; Gallese \& Lakoff, 2005). For example, the neural structures involved in sensory, perceptual, or motor information processing are also active when processing words whose meaning embeds prominent sensory (Goldberg, Perfetti \& Schneider, 2006), perceptual (Aziz-Zadeh et al., 2008; Martin et al., 1995) or motor features (Heil et al., 1999; Macedonia, Müller, \& Friederici, 2011; Masumoto et al., 2006; Nilsson et al., 2000; Nyberg et al., 2001). Moreover, different linguistic tasks in fMRI studies involved a somatotopic activation of the premotor and primary motor cortices, both in children (James \& Maouene, 2009) and in adults (Aziz-Zadeh et al., 2006; Hauk, Johnsrude, \& Pulvermuller, 2004; Tettamanti et al., 2005), pointing to the embodied nature of language.

In line with this evidence, motor enrichment seems to play an especially important role in language learning. Children seem to benefit from motor enactment, both in native language acquisition and foreign language learning; 11 months old babies' spontaneous pointing behaviors predict later verbal development of their native language (Acredolo \& Goodwyn, 1988), and 5 year old children seem to benefit more from motor (iconic gestures performed in the learning stage) than from visual enrichment in foreign language vocabulary learning (Tellier, 2008). In adults, self performed iconic gestures improve learning more than visual enrichment or verbal learning (Mayer et al., 2015), and more than irrelevant gestures (Kelly, McDevitt \& Esch, 2009; Macedonia \& Knösche, 2011). Lastly, adding gestures to the learning process seems to broaden the neural representation of new vocabulary, and activate the premotor cortex during a word-picture matching task (Macedonia, Müller, \& Friederici, 2010). Iconic gestures were also shown to assist in lexical retrieval, i.e., in the recall of words (Krauss \& Hadar, 1999).

This line of research suggests that second or foreign language learning is more efficient when it takes place in a naturalistic context, one that involves as much interaction of the body with its surroundings as possible. In these conditions, the body can serve as a potent tool in the learning process and improve it significantly (Macedonia, Kern, \& Roithmayr, 2014). While sensory enrichment (pictures, audio files, short videos etc.) is common in today's foreign language classroom, motor enactment is generally absent from it. When used, motor enactment is limited to pairing new vocabulary items (in text or sound) to symbolic (or iconic) gestures, either self generated or imitated. Although such enrichment was found to improve long term memory of new vocabulary (e.g. Macedonia et al., 2014), it remains purely symbolic, and does 
not offer a true-to-life sensory-motor integrative experience that would mimic learning in the wild'. When we learn a new word in our mothers tongue or in a foreign language, it is imbued in rich, sensory-motor and socio-cultural context. A 'flower' is not only its shape, color, smell and touch, but also the motor-actions that it affords when we interact with it (reaching out and picking it, smelling it, etc.).

In this work, we introduce a new paradigm to foreign language learning using Virtual Reality (VR) technology. We suggest that this technology may offer a unique opportunity to integrate learning in a rich sensory-motor context and 'bring back the body' into the language learning classroom.

Virtual reality generally refers to "a simulated environment whose reality content, and optionally other senses, is entirely produced by computer, and the appearance of the environment alters with the participant's behavior" (Rebenitsch \& Owen, 2016). VR is a combination of technological devices that allow the user to investigate, create and interact with a 3D environment. Its immersive nature provides a real-life experience that is more engaging than imaginal exposure (Riva, 2011). The potential similarity of the virtual world to the real world relies on three main ingredients: sight, hearing, and interaction (Repetto, 2013). First, visual stimulation is provided mostly via a computer monitor or a head-mounted-display (HMD). HMD is a visualization helmet, displaying computer-generated images to both eyes, possibly inducing a 3D illusion of the virtual environment and increasing its realism. The experience with HMD is defined "immersive" because the user is both embedded in the virtual world and completely isolated from the real world. Second, auditory devices can be used, such as head-based headphones or stand-alone speakers. Third, the interaction relies mostly on the capabilities offered by the software - the more the users see their actions influence the virtual world, the more they feel engaged and immersed. These three qualities of the VR experience increase the sense of presence perceived by the user (Lombard \& Ditton, 1997). Presence is "the non-mediated (pre-reflexive) perception of using the body/a medium to successfully transform intentions in action (enaction)" (Riva, 2011). Enhanced sense of presence in the VR is congruent with embodied cognition theories (Shapiro, 2011). Therefore, VR can be considered an "embodied technology" for its effects on body perceptions (Riva, 2002).

Together with the sense of presence, other features of VR can facilitate and enhance learning processes. First, continuous perceptual-motor loop in VR reflects the ongoing process of real-time action-based perception, which changes dynamically as the user moves through and interacts with the virtual environment in real-time (Riva, Davide, \& ljsselsteijn, 2003). Also, VR studies show that users identify a virtual avatar's body as their own and exhibit perceptual biases related to body features (Blanke, Slater, \& Serino, 2015; Slater, Perez-Marcos, Ehrsson \& Sanchez-Vives, 2009; Slater et al., 2010). Altogether, the perceptual-motor loops and the virtual body identification demonstrate VR's ability to facilitate temporary sensory remapping by affecting multimodal integration processes, such as synchronized visual, proprioceptive, and vestibular processing. In line with that concept, multimodal processing was shown to have shorter reaction times over the processing of unimodal and bimodal stimuli (Hecht, Reiner \& 
Halevy, 2006). Such enhanced virtual multimodal stimuli efficiency allows the user to better allocate attention to details of the stimuli, which may be of great importance in the context of learning new content. Moreover, learning new tasks from a 2D instructor display (e.g., computer screen) were shown to result in a higher cognitive load compared to a 3D projection of the same instructor as a digital avatar (Dan \& Reiner, 2017). Altogether, these findings indicate that a virtual reality environment can support efficient learning processes.

Furthermore, as an embodied technology, VR seems to be a promising tool to investigate the link between language and action. As established before, performing a meaningful action together with new vocabulary learning improves memory for the novel words. Within the VR setting, actions accompanying new vocabulary can be demonstrated by avatars, virtual agents, as effectively as by human instructors.

Macedonia et al. (2014) found that learners memorized new vocabulary better when they performed an iconic gesture associated with the new word than when they merely watched an agent (real or virtual) performing the gesture. As previously stated, a direct interaction may be more beneficial for vocabulary learning than iconic gestures, as it simultaneously activates representations of the word's ecological affordance. Such direct interaction can be performed using VR technology, which provides a natural yet controlled setting with much lower stakes than encountered in the real world. Unlike learning, active as it may be, in the classroom or field, VR technology makes experimenting with different 'what if' scenarios possible, with little or no consequences (e.g., lighting a fire, dropping objects, or lifting heavy equipment).

This study aims to explore the effect of meaningful motor information in VR setting on learning new words in an unfamiliar language. Vocabulary acquisition is a significant challenge in foreign language learning, and in order to test whether meaningful motor output accompanying speech influences new vocabulary acquisition, we explored the effect of direct motor interaction with objects in VR setting on the memory of their names in a foreign language. To that extent, participants used a 3D virtual reality head-mounted display, which allows the replacement of the natural visual and auditory environment with computer-generated 360 degrees content. Participants experienced a VR environment containing everyday objects to test the contribution of sensory input and motor output on remembering the objects' names in the foreign language Finnish. We tested the influence of the user's mode of interaction with specific everyday objects in the virtual world on the memorization of the objects' Finnish names. We specifically hypothesize that direct meaningful motor interaction with objects in VR setting will improve memory for the objects' names in Finnish, more than non-meaningful motor output or no motor output at all. 


\section{Methods and Materials}

\section{Participants}

Forty-six participants (22 males and 22 females, age: range $=19-41$ years, $M=28.41, S D=$ 4.94) were recruited for the experiment through advertisements in social media networks. All participants were right-handed and had normal or corrected to normal vision. All participants were native Hebrew speakers with no proficiency in Finnish (according to self-report in a standard language experience questionnaire). Sixteen participants volunteered to participate in the study, and 30 participants were paid to do so (no significant difference was found between these two groups in any of the dependent measures of the experiment). Data of one participant were discarded from analysis due to below chance-level performance (25\%).

\section{Stimuli}

Finnish was selected as the training language to rule out previous experience or familiarity with the new vocabulary. Finnish is a language that relatively few Israelis are proficient in or exposed to regularly. Moreover, its phonetic structure does not resemble the ones of Germanic, Semitic, or Romance languages that are widespread in Israel. Forty nouns describing daily objects in the Finnish language were selected (see Appendix A for the full list and Figure 1 for examples). The vocabulary set was standardized for syllable length (range: 2-3 syllables). Vocabulary items were recorded by a native Finnish-speaking woman, and cut into single-word audio files $( \pm 1$ second long each).

Items were pre-tested for identification, familiarity, and common use. In a questionnaire pre-test, 19 Israeli students labeled each object, depicted in a 2D picture cut out of the experiment's virtual environment. Then, they were asked to rate each object on a 1 to 7 familiarity scale. Finally, participants stated the most frequent action associated with each object (for example: broom - sweeping, pen - writing).

Object identification was correct in $97 \%$ of cases on average ( $S D=15 \%)$, and the average rated level of the objects' familiarity was $5(S D=0.98)$. Two independent judges reviewed the relevant actions participants listed for each object, and chose the most frequent concrete one. For example: for the object 'ball' the verb 'play' was a more frequent response (14 times), but 'throw' (listed 4 times only) was chosen for its concreteness and straightforwardness. In total, the average level of compliance between the relevant action that was listed and the one chosen was $78 \%(S D=41 \%)$.

For each object, specific relevant hand action and irrelevant hand action were recorded, while executed by a virtual instructor, as a moving avatar figure in the virtual environment. All recorded movements were identical in the length of display. 
Participants learned the new vocabulary items in an inter-subject block design under three conditions (The complete set of manipulation and irrelevant movements is listed in Table 1 in the Appendix, and some of them are demonstrated in the supplementary video 1):

1. Watch-only: participants repeated the object's name while merely watching it in context, without performing any motor action (see video example 1).

2. Irrelevant movement: participants repeated the object's name while performing an irrelevant, non-interactive movement, modelled by an avatar. The modelled trajectories were designed as geometric forms (e.g., tracing half a circle with both hands simultaneously). These irrelevant actions were bare of any distinct iconic or symbolic image that could be associated with the word meaning (see video example 2).

3. Manipulation movement: participants repeated the object's name while performing a meaningful interaction with it, modelled by an avatar. These movements mimicked functional actions performed in reality to manipulate the specific object (e.g., gripping the virtual hat, lifting it and putting it on one's head) (See video example 3).

Each participant learned 13 or 14 different words in each learning condition. The items were counterbalanced across the learning conditions considering the semantics and phonological properties of each target word (number of syllables and syllabic structure). Considering this distribution, three different orders of objects were semi-randomly costumed, and all possible orders of the three learning conditions were implemented in the experiment, to which participants were randomly assigned.

\section{Virtual environment}

The virtual environment, developed by the Center for Educational Technologies (CET), was an immersive virtual room (Fig. 1). This content was displayed using an Intel(R) Core(TM) i7-6700K processor using Unity 5.6 software and an HTC-Vive headset. The participant stood at the centre of the laboratory room and was outfitted with the headset. The participant controlled his navigation within the virtual environment by walking, precisely as he would in the real world. The borders of the laboratory room were marked in the virtual environment as a blue net, which the participant was instructed not to pass during his navigation.

When entering the virtual environment, the participant started from an exterior virtual environment as a training area, where s/he stood outdoors under clear sunny blue skies. The ground was covered by green grass and surrounded by trees and buildings. No human being was virtually present in the scene, and three training objects were scattered across the grass 
(camera, television, kite). Once completing the training session according to the experimental condition, the participant was automatically transferred to the virtual room where the experiment took place. This was a four-walled room, containing a door and two windows, in which several items were kept at all times (a table, a chair, a chest of drawers, two shelves, a mirror, a kitchen surface, a sink, an oven, and two wall pictures), while the experimental items changed between the different blocks. Although the virtual objects were not solid entities and could be passed through by the participant, they contained physical properties and could not be overlapped in position with other objects.

The participant her/himself was present in the virtual environment as an avatar, consisting of head and hands. A pair of controllers held by the participant throughout the experiment, one in each hand, served as a reference for the VR system, which allowed for real-time tracking of the participant's hands. The controllers contained two functional buttons (Fig. 2B). The top button was used to point or to activate an object, depending on the experimental condition. The lower button was pressed in order to form a fist with the virtual hand or to grab an object, depending on the experimental condition.

\section{Word learning assessment}

Following each learning block, subjects performed a computerized word-picture matching test (using the software OpenSesame 3.1.9). The participants heard one of the Finnish words to which they had been exposed in the learning session and selected the corresponding item out of four 2D object pictures. The distractor pictures (modelled after PPVT-4: Peabody picture vocabulary test, Dunn \& Dunn, 2007) included one item of the same semantic category as the target item, a phonologically similar item, and one item that was included in the virtual environment whose name was not studied as a Finnish word. The test was comprised of all the Finnish words learned in the antecedent session, displayed in randomized order. Following their response, participants ranked their confidence level on a 1-7 scale.

To assess long-term lexical learning effects, participants repeated the word-picture matching test a week after the initial learning session. In addition, a free word recall test was administered before the second word-picture matching test alone, to avoid possible interference with the word-picture matching test results. In the free recall test, participants were asked to retrieve as many of the Finnish words they learned in the previous week as they could remember, together with their Hebrew translation. The answers were scored in two separate ranking dimensions by two judges blind to the experimental learning condition of the recalled words. A ranking of $0,0.5$, or 1 was given to the level of accuracy of the recalled Finnish words. Fully recalled Finnish words were ranked with the score 1, partially recalled Finnish words (including minor omissions or substitutions) were ranked with the score 0.5 , and incorrectly recalled or not recalled words were ranked with a score of 0 . Importantly, Hebrew-sounding pronunciation of the Finnish words was not considered an error in the rating scheme. The second ranking dimension was given to the recalled Hebrew translation. This ranking was given only when the Finnish word was fully or 
partially retrieved. Correct translation was ranked with the score 1, a partially correct translation was ranked with the score 0.5 , and incorrect translation was ranked with the score 0 . The inter-rater reliability of the recalled Finnish words was $r=0.90(p<.05)$ and the inter-rater reliability of the recalled Hebrew translation was $r=0.76(p<.05)$. Only items that got $100 \%$ interrater agreement were included in the analysis.

\section{Procedure}

During the experimental protocol, the participants were welcomed in a quiet room by the experimenter. After reading and signing an informed consent form, the participants filled their demographics in an online form. Then, the experimental task started. Each participant was randomly assigned to one of the six orders of the experimental conditions (see supplementary video).

Before every experimental block, instructions on how to use the VR equipment (controllers and headset) and what to do in the virtual environment were given to the participant by the experimenter. Thereafter, the participant wore the headset with the headphones and held the controllers, while the experimenter launched the practice session in order to let the participant familiarise with the environment and interact with it. After each practice session, which was congruent with the experimental condition, the participant was asked to stand in a virtual round frame/ spotlight which was marked on the ground and face the direction of an arrow which was placed next to the frame/ spotlight. Then, the participant was informed that $s / h e$ will be automatically transferred to the environment and was asked if s/he is ready to do so. Once the consent was given, the participant was virtually transmitted to the simulated room where the experimental task took place.

The main task was to learn 13 or 14 object's names in Finnish in one learning session. The virtual room where the learning session took place contained all the objects to be learned in that session. There were three learning sessions in total, in a block design according to the three learning conditions.

In the Watch-only learning condition, the participant located a white spotlight on the ground of the virtual environment, with an arrow mark placed next to it. The participant walked and stood inside the frame/ spotlight, which then changed its colour to green. According to the arrow's direction, the participant located an object marked with a yellow outline. Once the object was located, the participant heard the Finnish word describing the object, and repeated the word out loud. Then, the participant walked towards the object to look at it closely, returned to stand in the frame, heard the Finnish word describing the object for the second time and repeated it aloud again. Once the participant finished repeating the word for the second time, the object's yellow outline and the matching frame glowed together with an auditory cue around a new object. This way, all 13 or 14 objects' names were learned by the participant. During the 
watch-only condition, the participant was instructed to hold the controllers, but not to perform any hand movement when observing the object.

This procedure was identical in the two other learning conditions, with the only difference in the occurrence between the two repetitions on the Finnish word. In both the Manipulation movement and the Irrelevant movement conditions, after the participant heard and repeated the object's name in Finnish, an avatar appeared next to the marked object, demonstrated a movement, and then disappeared. The participant watched the avatar as the movement was demonstrated, and once the avatar disappeared, the participant walked to stand at the same position as the avatar and repeated the movement, as demonstrated. When finished, the participant walked back to the frame, to hear and repeat the object's name for the second time. Whereas the movement executed in the Manipulation movement learning condition referred to the object and involved touching, moving, or activating the object, the movement in the Irrelevant movement learning condition did not. In both conditions, the participant used the controllers to execute the movements, and pressed the controllers' functional buttons to produce specific hand actions, as described above. In the Manipulation movement condition, participants were instructed to approach and virtually manipulate the target objects, although the avatar had not done so in the demonstration section.

After each of the three learning sessions, the participant handed the controllers to the experimenter and was assisted by her to remove the headset and headphones. Then, the participant sat in front of a computer, different from the VR computer, to perform the word-picture matching test. The meeting lasted for about an hour, in which each learning block lasted for approximately $15 \mathrm{~min}$, and each word-picture matching test for about $3 \mathrm{~min}$. After the third word-picture matching test following the third learning block, the participants were asked to fill out an online review form about their learning experience, after which the first meeting of the experiment was over.

Participants returned for a follow-up meeting precisely one week after the first meeting. Within the second meeting, which lasted about $10 \mathrm{~min}$, participants completed the free recall test and then repeated the word-picture matching test, this time completing the three different blocks one after the other. In this part of the experiment, participants were not required to wear the HTC headset or perform any actions in the virtual environment.

\section{Statistical analysis}

For the word-picture matching test data, we used a $2 \times 3$ factorial design with the within-subject factors of Learning Condition (Manipulation movement, Irrelevant movement, and Watch-only) and the Timing of the test (day 1 or day 8). For the free recall test data, a factorial design was used, only for the Learning Condition factor, as the test was administered only on the second time point. A separate analysis was conducted for each of the recalled dimensions: the recalled Finnish pronunciation and the recalled translation of the recalled Finnish words. 
Repeated measures analysis of variance (ANOVA) was conducted for both the word-picture matching and free recall tests, with a predetermined significance level of 0.05 . Post-hoc testing of the hypothesis that the word-picture matching accuracy, reaction time and recall accuracy in the Manipulation movement condition were improved relative to the two other conditions was conducted following each of the ANOVAs using one-sided t-tests that compared specific pairs of learning conditions.

Since the normality assumption for the factor Learning Condition was not met as indicated by Kolmogorov-Smirnov test, we adopted a bootstrapping technique (resampling with replacement), which provides estimates that do not rely on the assumption of normality (Bishara \& Hittner, 2012). In each iteration, a Learning Condition label (Manipulation movement, Irrelevant movement, Watch only) was randomly assigned to accuracy or reaction time data. Then, we conducted a two-way ANOVA, one-way ANOVA, and Student's t-test in the cases of word-picture matching, recall, and post-hoc tests, respectively, similarly to the original tests in each of these comparisons. This step was repeated 10,000 to generate a background distributions of $\mathrm{F}$ scores of the resampled data in the case of ANOVA, and $t$ scores in the case of the post-hoc tests. For the effect of the interaction between the Learning Condition and the Time of the test, the resampling condition was conducted separately in each one of the two time points, before the $\mathrm{F}$ score of the tossing was determined.

Finally, The $\mathrm{F}$ and $\mathrm{t}$ scores obtained in the original ANOVA and post-hoc t-tests were compared to the background distributions, and thus the p-values were determined for each of the tests. Benjamini-Hochberg procedure (to reduce false discovery rate, FDR) for dependent data (Benjamini \& Hochberg, 1995) was performed to correct for multiple comparisons.

\section{Results}

The reported results refer to two testing sessions: day 1 (immediately after each learning block) and day 8 ( 1 week after the learning session). In addition, they refer to two testing modes: computerized word-picture matching test and verbal free recall test (performed only on day 8). The recalled items were transcribed by the experimenter and were rated by two independent raters for pronunciation and translation accuracy $(0 / 0.5 / 1)$.

\section{Vocabulary word-picture matching task}

To assess the influence of Time (day 1 , day 8 ) and Learning Condition (Manipulation movement, Irrelevant movement, Watch only) on the accuracy of the word-picture matching of pictures depicting the learned Finnish vocabulary, we conducted a two-way repeated measures ANOVA (Figure 3).

The Learning Condition yielded a main effect on word-picture matching accuracy $(F(2,88)=$ $4.81, p=.01)$. A strong main effect of Time was obtained $(F(1,44)=126.46, p<.0001)$, revealing that the performance was higher in the first word-picture matching test. We also 
obtained a significant Time $\times$ Learning Condition interaction effect on word-picture matching accuracy $(F(2,88)=4.66, p=.01)$.

At the first time point, the word-picture matching accuracies in the Manipulation movement, Irrelevant movement, and Watch only conditions were $75 \% \pm 2 \%, 67 \% \pm 22 \%$, and $78 \% \pm 16 \%$ (mean \pm standard deviation), respectively. At the second time point, the accuracies were $59 \% \pm 19 \%, 51 \% \pm 19 \%$, and $54 \% \pm 17 \%$, respectively. Post-hoc one-sided analyses indicated that the word-picture matching accuracy was significantly higher in the Manipulation movement relative to the Irrelevant movement conditions at both time point $1\left(t(44)=2.51, p=.007, Q_{\mathrm{FDR}}<\right.$ $.05)$ and time point $2\left(t(44)=2.22, p=.015, Q_{\mathrm{FDR}}<.05\right)$. At time point 2 , we also found that the accuracy in the Manipulation movement condition was marginally higher than in the Watch-only condition $\left(t(44)=1.76, p=0.04, Q_{\mathrm{FDR}}<.06\right)$. The accuracy in the Manipulation movement condition was not significantly higher relative to the Watch-only condition at time point $1(t(44)=$ $1, p=.84)$.

We further assessed the effect of Time and the Learning Condition over the participants' reaction time (defined as the time interval between the onset of the playback of the Finnish word and the participant's response) using a two-way repeated measures ANOVA with the same two factors (Figure 4). At the first time point, the reaction times in the Manipulation movement, Irrelevant movement, and Watch-only conditions were $3.85 \pm 1.22,3.94 \pm 1.23$, and $3.83 \pm 1.07$ seconds, respectively. At the second time point the reaction times were $4.00 \pm 1.22,4.24 \pm 1.27$, $4.17 \pm 1.40$ seconds, respectively. No effect was found for the Time of test $(F(1,43)=2.71, p=$ $0.11)$, the Learning Condition $(F(1,43)=0.37, p=.52)$, or the interaction between them $(F(2,86)$ $=0.42, p=.68)$.

\section{Vocabulary recall}

To assess the influence of the Learning Condition on the long-term memory retrieval of the new vocabulary items (i.e., recall in time point 2), we conducted a one-way repeated measures ANOVA comparing recall accuracy between the conditions Manipulation movement, Irrelevant movement, and Watch-only (Figure 3). For the dimension of the free recalled Hebrew translation of the recalled Finnish words (Figure 5a), the participants accurately recalled $0.41 \pm 0.47$, $0.16 \pm 0.33$, and $0.22 \pm 0.38$ words in the Manipulation movement, Irrelevant movement, and Watch-only conditions, respectively. A significant main effect of the Learning Condition on recall accuracy was observed $(F(2,72)=4.55, p=.01)$. Post hoc analyses indicated that the participants recalled the meaning of the word more accurately if they had learned it under the Manipulation movement condition relative to both Watch-only $\left(t(43)=1.99, p=.026, Q_{\mathrm{FDR}}<.05\right)$ and Irrelevant movement $\left(\mathrm{t}(43)=3.02, p=.002, Q_{F D R}<.05\right)$ conditions.

For the dimension of free recalled pronunciation of the learned Finnish words (Figure 5), we found a similar significant main effect $(F(2,72)=3.38, p=.04)$. The participants accurately recalled $0.37 \pm 0.43,0.17 \pm 0.30$, and $0.28 \pm 0.40$ words in the Manipulation movement, Irrelevant movement, and Watch-only conditions, respectively. The pronunciation was more accurate for words learned under the Manipulation movement condition relative to the Irrelevant movement 
condition $\left(t(43)=2.73, p=.003, \mathrm{Q}_{\mathrm{FDR}}<0.05\right)$, but not relative to the Watch-only condition $(t(43)$ $=1.07, p=.14)$.

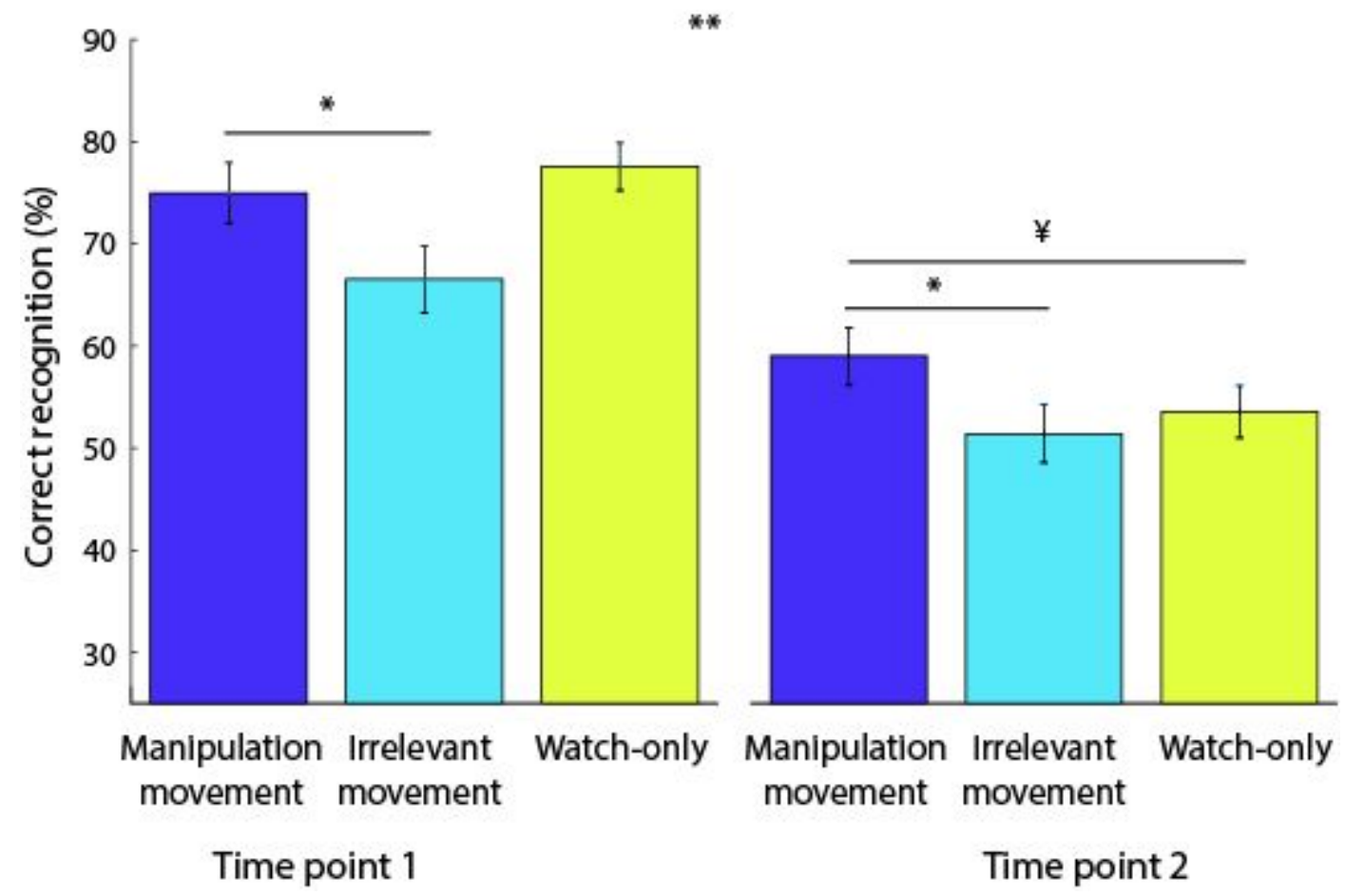

Figure 3: word-picture matching accuracy of learned Finnish words by Learning Condition and Time.

${ }^{*} p<.05, Q_{F D R}<.05 ;{ }^{*} p<<.0001 ; ¥ p<.05, Q_{F D R}<.06$. 
Time point 1

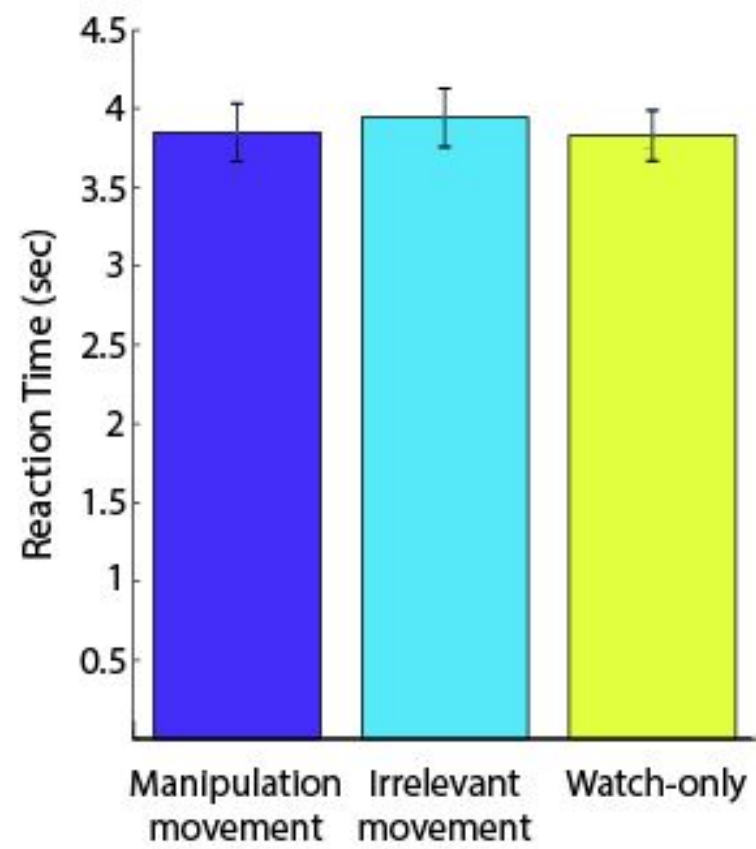

Time point 2

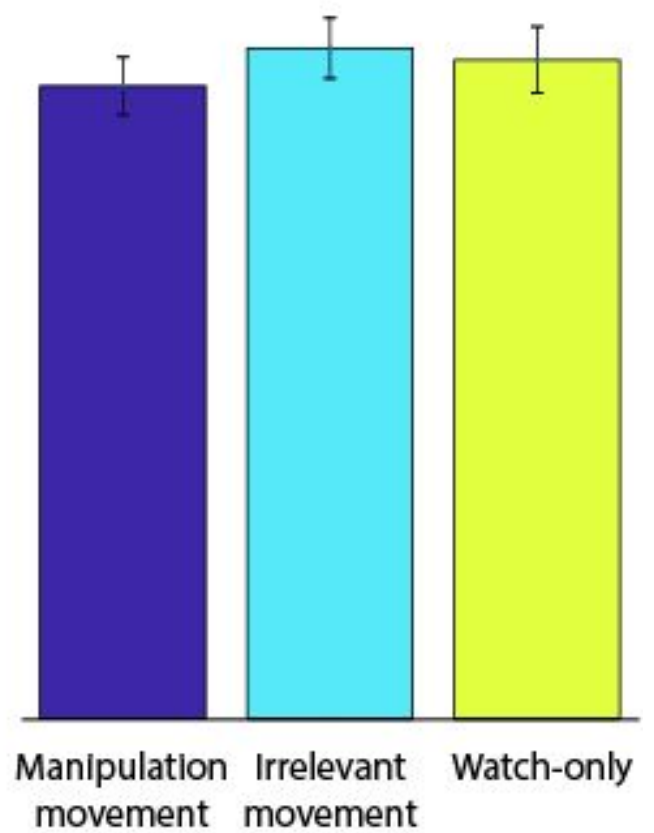

Learning Condition

Figure 4: Reaction times in the word-picture matching test by Learning Condition and Time

Figure 5: Long-term recall of Finnish words in terms of translation and pronunciation (day 8 ) ${ }^{*} p<.05, Q_{F D R}<.05 ;{ }^{* *} p<.005, Q_{F D R}<.05$.

\section{Discussion}

The present study is an initial investigation into the unique benefits of virtual reality as a tool for foreign language learning. This work demonstrates one of VR's most prominent advantages: the opportunity to offer true-to-life interaction with 3-D simulated objects, providing rich and integrated sensory and motor input that may promote vocabulary learning. Overall, the results of this first study indicate that adding natural and affordable motor interaction with objects in a VR setting significantly improves vocabulary learning in a foreign language.

A close look at the results reveals that performance in the word-picture matching task was better for items that were paired with a manipulation movement: a relevant action of interaction with the object, than for items that were paired with an irrelevant action that is not afforded by our knowledge about the object and our natural interaction with it. This was true in both recognition tests, during the learning session (time 1) and one week later (time 2). The results concur with our prediction, pointing to a facilitation effect of manipulation action which cannot be explained by general, irrelevant, motor activity. It may also be the case that repeating the Avatar's movements in both active conditions, irrelevant action and manipulation action, put a cognitive load on the learning process. Participants had to attend to the movements, remember and execute them as close as possible to the original demonstration. In the irrelevant action condition the action was comprised of several hand movements that could not have been easily labeled with a verb and thus might have put a higher cognitive load on the learning process.

However, the manipulation action condition was favourable to the Watch-only condition only at time 2, which raises some questions that may need to be disambiguated in later work. First, the similar results in the Watch-only and manipulation action conditions on time 1 do not replicate previous findings, pointing to a significant effect of iconic gestures relative to a viewing condition (e.g, Macedonia \& Knösche, 2011; Tellier, 2008). It may be the case that the immersive 3-D VR technology in our study enhanced learning in both conditions; both learning in the Watch-only condition and memory traces for word-picture matching in time 1. A possible explanation might be, that performance in the word-picture matching task was generally high during the learning session (time 1) on both the manipulation action and the watch-only conditions. In this case, the Manipulation movement effect might have been 'wiped out' by the generally high performance in the word-picture matching rate on time 1. However, the memory of the new vocabulary may have decayed substantially in the follow-up test, a week later. Then, participants possibly had to make more effort to actively recall the new vocabulary and the variance was exposed. In this case, the motor information that was paired with the new vocabulary in the Manipulation action condition added more information and might have served as a more powerful mnemonic. 
As can be gleaned from the results, performance in the irrelevant action condition was lower than in the Watch-only condition and the Manipulation movement condition, on both testing times. These results are consistent with previous findings that point to an interference effect for irrelevant or meaningless actions, that can explain the lower performance in this condition (e.g, Rumiati \& Tessari, 2002). Anecdotally, participants commented about the difficulty to repeat the Avatar's movements in the irrelevant condition, as they were unnatural and not easy to remember. This may have created additional cognitive load and attracted attention that stood in the way to effective learning of the new vocabulary. Namely, it may be that movement during vocabulary learning is costly in cognitive resources. When this costly movement is directed towards enriching the motor representation of the object to be named, the benefit is equal or larger than the cost, and object manipulation therefore contributes to the object naming. Irrelevant motion, in contrast, has only the cost but not the benefit (or not enough benefit) for vocabulary learning, and therefore in most cases it showed even lower learning than the no-movement, Watch only condition.

In general, the performance in the free recall test (administered only on time 2) was poor, with less than one word on average recalled correctly out of 14 in each learning condition (compared to over 5 words on the time 2 word-picture matching test). These results correspond with the typical performance advantage in word-picture matching over recall tests, evidenced in the memory research literature (e.g., Tulving, 1976).

Despite the low recall rate, recalling the meaning of the words that were paired with a Manipulation movement was better than the meaning of the words that were learned in the two other conditions. We found this advantage in both recall dimensions: pronunciation (in Finnish) and translation (to Hebrew). Again, these results point to a manipulation movement effect when task demands are high; the advantage of a richer sensorimotor context in terms of recall is revealed when the long term memory is challenged by the passage of time.

As to date, foreign language instruction is still largely based on amodal methodologies, such as drilling of bilingual lists (a word in the target language and its translation). This is contrary to the growing body of evidence pointing to the benefits of sensory motor learning.

Reasons for the present state are varied. Implementing findings of scientific studies to transform pedagogical practices is often a long and complex process, one that requires a paradigm shift as well as considerable resources. Among other challenges, most traditional class scenarios limit the range and authenticity of motor enactment as a learning paradigm. Thus, attempts to incorporate motor enactment into the curriculum are reduced to iconic and deictic gestures (e.g, pointing) to symbolize an aspect of a word's meaning. While iconic gestures are indeed symbolic in nature, they nevertheless lack some key aspects of natural motor interaction. Importantly, manipulation movement maintains a link between the motor action and its function in the physical world and therefore unlike iconic gestures, it does not short-circuit the 
perception-action loop. However, the generation of such realistic context is even harder to achieve in a classroom setting.

To conclude, this study examined the potential benefits of direct motor interaction with objects in VR setting on the memory of their names in a foreign language, compared to meaningless, unrelated movement or watching the objects with no movement execution. This study shows that motor interaction with objects in a VR setting promotes better memory of novel vocabulary items in the long term, compared to items learned with no movement, but not compared to watching the objects in the VR context with no movement execution. Further studies should be conducted to explore the effect of mere exposure in VR setting to the presence of the novel vocabulary physical illustration on the memory of the newly acquired words. Also, combining imaging or neurophysiological methods is necessary to deepen the understanding of the learning processes revolving enactment in a VR setting.

\section{Acknowledgements}

We thank the Minducate Research and Innovation Center for Science of Learning at the Sagol School of Neuroscience (TAU) for their generous support and sponsorship.

We thank the Center for Educational Technology for fruitful collaboration in the characterisation and development of the virtual reality environment used in this experiment. Specifically, we would like to thank the ReCET innovation lab members: Ofer Tiber, Danny Zavaro, Idan Yitzhaki and Yoel Trubek for their creative hard work.

We would also like to thank Talma Hendler, the head of the Sagol Brain Institute for access to the XR lab facilities. 


\section{References}

Acredolo, L., \& Goodwyn, S. (1988). Symbolic gesturing in normal infants. Child Development, 59, 450-466.

Aziz-Zadeh, L., Fiebach, C. J., Naranayan, S., Feldman, J., Dodge, E., \& Ivry, R. B. (2008).

Modulation of the FFA and PPA by language related to faces and places. Social

Neuroscience, 3(3-4), 229-238.

Aziz-Zadeh, L., Wilson, S. M., Rizzolatti, G., \& lacoboni, M. (2006). Congruent embodied representations for visually presented actions and linguistic phrases describing actions.

Current Biology, 16(18), 1818-1823.

Barsalou, L. W. (2008). Grounded cognition. Annual Review of Psychology, 59, 617-645.

Benjamini, Y., \& Hochberg, Y. (1995). Controlling the false discovery rate: a practical and powerful approach to multiple testing. Journal of the Royal Statistical Society: series B (Methodological), 57(1), 289-300.

Blanke, O., Slater, M., \& Serino, A. (2015). Behavioral, neural, and computational principles of bodily self-consciousness. Neuron, 88(1), 145-166.

Dan, A., \& Reiner, M. (2017). EEG-based cognitive load of processing events in 3D virtual worlds is lower than processing events in 2D displays. International Journal of Psychophysiology, 122, 75-84.

Dunn, L. M., \& Dunn, D. M. (2007). PPVT-4: Peabody picture vocabulary test. Pearson Assessments.

Gallese, V., \& Lakoff, G. (2005). The brain's concepts: The role of the sensory-motor system in conceptual knowledge. Cognitive Neuropsychology, 22(3-4), 455-479.

Goldberg, R. F., Perfetti, C. A., \& Schneider, W. (2006). Perceptual knowledge retrieval activates sensory brain regions. Journal of Neuroscience, 26(18), 4917-4921.

Hauk, O., Johnsrude, I., \& Pulvermüller, F. (2004). Somatotopic representation of action words in human motor and premotor cortex. Neuron, 41(2), 301-307.

Hecht, D., Reiner, M., \& Halevy, G. (2006). Multimodal virtual environments: response times, attention, and presence. Presence: Teleoperators and Virtual Environments, 15(5), 515-523.

Heil, M., Rolke, B., Engelkamp, J., Rosler, F., Ozcan, M., \& Hennighausen, E. (1999). Eventrelated brain potentials during recognition of ordinary and bizarre action phrases following verbal and subject-performed encoding conditions. European Journal of Cognitive Psychology, 11(2), 261-280. 
James, K. H., \& Maouene, J. (2009). Auditory verb perception recruits motor systems in the developing brain: an fMRI investigation. Developmental Science, 12(6), F26-F34.

Kelly, S. D., McDevitt, T., \& Esch, M. (2009). Brief training with co-speech gesture lends a hand to word learning in a foreign language. Language and Cognitive Processes, 24(2), 313334.

Krauss, R. M., \& Hadar, U. (1999). The role of speech-related arm/hand gestures in word retrieval. In R. Campbell \& L. Messing (Eds.), Gesture, speech, and sign (93-116). Oxford: Oxford University Press.

Lombard, M., \& Ditton, T. (1997). At the heart of it all: The concept of presence. Journal of Computer-Mediated Communication, 3(2).

Macedonia, M. (2014). Bringing back the body into the mind: gestures enhance word learning in foreign language. Frontiers in Psychology, 5, 1467.

Macedonia, M., \& Knösche, T. R. (2011). Body in mind: How gestures empower foreign language learning. Mind, Brain, and Education, 5(4), 196-211.

Macedonia, M., Kern, R., \& Roithmayr, F. (2014). Do children accept virtual agents as foreign language trainers?. International Journal of Learning, Teaching and Educational Research, 7(1).

Macedonia, M., Müller, K., \& Friederici, A. D. (2010). Neural correlates of high performance in foreign language vocabulary learning. Mind, Brain, and Education, 4(3), 125-134.

Macedonia, M., Müller, K., \& Friederici, A. D. (2011). The impact of iconic gestures on foreign language word learning and its neural substrate. Human Brain Mapping, 32(6), 982998.

Masumoto, K., Yamaguchi, M., Sutani, K., Tsuneto, S., Fujita, A., \& Tonoike, M. (2006). Reactivation of physical motor information in the memory of action events. Brain Research, 1101(1), 102-109.

Martin, A., Haxby, J. V., Lalonde, F. M., Wiggs, C. L., \& Ungerleider, L. G. (1995). Discrete cortical regions associated with knowledge of color and knowledge of action. Science, 270(5233), 102-105.

Mayer, K. M., Yildiz, I. B., Macedonia, M., \& von Kriegstein, K. (2015). Visual and motor cortices differentially support the translation of foreign language words. Current Biology, 25(4), 530-535.

Nilsson, L. G., Nyberg, L., Klingberg, T., Åberg, C., Persson, J., \& Roland, P. E. (2000). Activity in motor areas while remembering action events. Neuroreport, 11(10), 2199-2201.

Nyberg, L., Petersson, K. M., Nilsson, L. G., Sandblom, J., Åberg, C., \& Ingvar, M. (2001). Reactivation of motor brain areas during explicit memory for actions. Neuroimage, 14, 521- 
528.

Paulus, M., Lindemann, O., \& Bekkering, H. (2009). Motor simulation in verbal knowledge acquisition. Quarterly Journal of Experimental Psychology, 62(12), 2298-2305.

Rebenitsch, L., \& Owen, C. (2016). Review on cybersickness in applications and visual displays. Virtual Reality, 20(2), 101-125.

Repetto, C. (2013). The relationships between language and action: the contribution of virtual reality in the domain of embodied cognition (Unpublished master's thesis).

Riva, G. (2002). Virtual reality for health care: the status of research. Cyberpsychology \& Behavior, 5(3), 219-225.

Riva, G. (2011). Presence, actions and emotions: A theoretical framework. Journal of CyberTherapy and Rehabilitation, 4(2), 204-206.

Riva, G., Davide, F., \& IJsselsteijn, W. A. (2003). Being there: The experience of presence in mediated environments. Being there: Concepts, effects and measurement of user presence in synthetic environments, 5 .

Rumiati, R., \& Tessari, A. (2002). Imitation of novel and well-known actions. Experimental Brain Research, 142(3), 425-433.

Slater, M., Spanlang, B., \& Corominas, D. (2010). Simulating virtual environments within virtual environments as the basis for a psychophysics of presence. In ACM Transactions on Graphics (TOG) (Vol. 29, No. 4, p. 92). ACM.

Slater, M., Pérez Marcos, D., Ehrsson, H., \& Sanchez-Vives, M. V. (2009). Inducing illusory ownership of a virtual body. Frontiers in Neuroscience, 3, 29.

Shapiro, L. (2011). New problems of philosophy. Embodied cognition. London: Routledge.

Tellier, M. (2008). The effect of gestures on second language memorisation by young children. Gesture, $8(2), 219-235$.

Tettamanti, M., Buccino, G., Saccuman, M. C., Gallese, V., Danna, M., Scifo, P., ... \& Perani, D. (2005). Listening to action-related sentences activates fronto-parietal motor circuits. Journal of Cognitive Neuroscience, 17(2), 273-281.

Tulving, E. (1976). Ecphoric processes in recall and recognition. In J. Brown (Ed.), Recall and recognition. New York: Wiley.

Figures and tables 
Figure 1: The two stage learning schedule (condition blocks were presented in counterbalanced order)

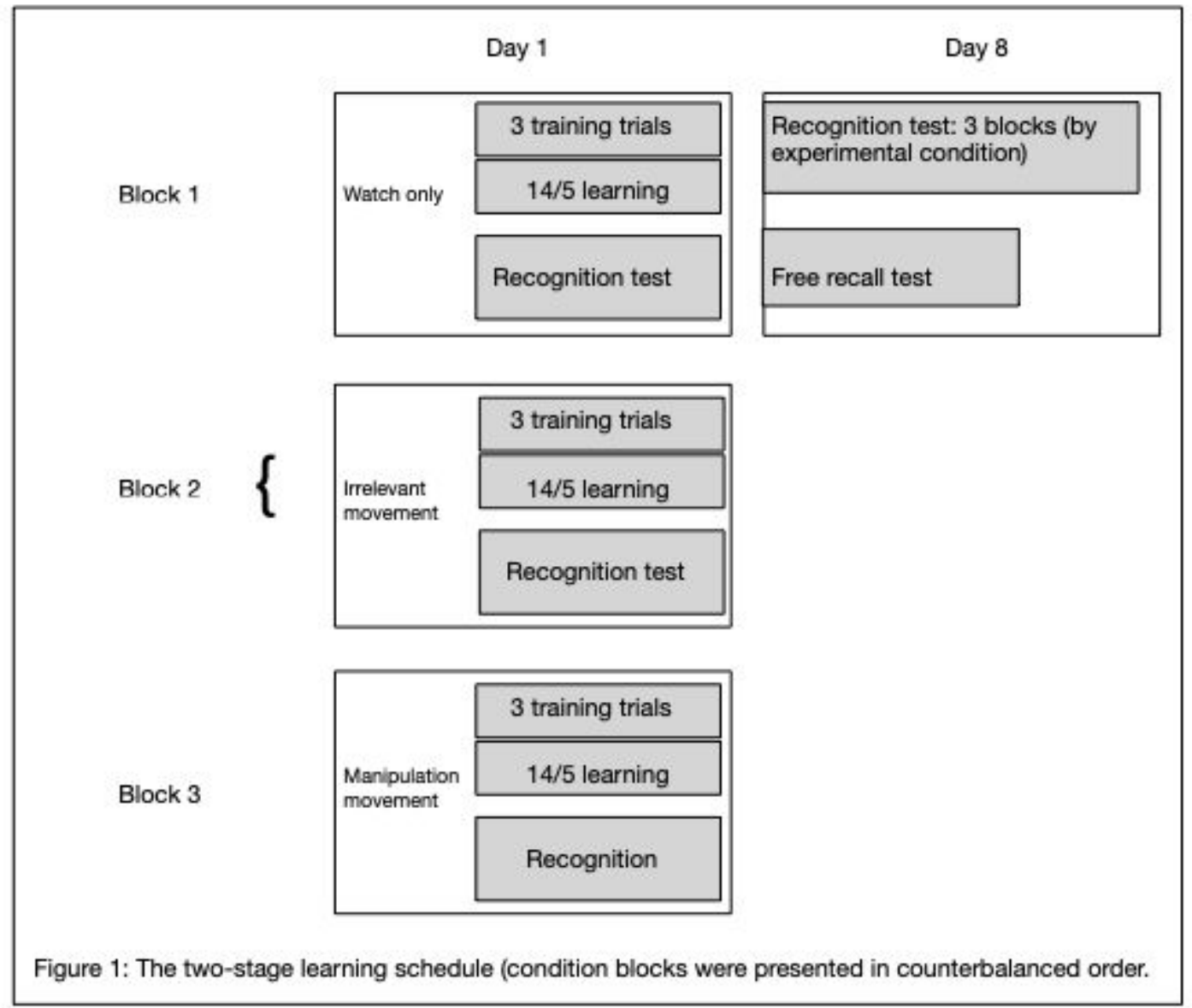


Figure 2: Manipulation movements with objects in the virtual environment (external viewer point of view)

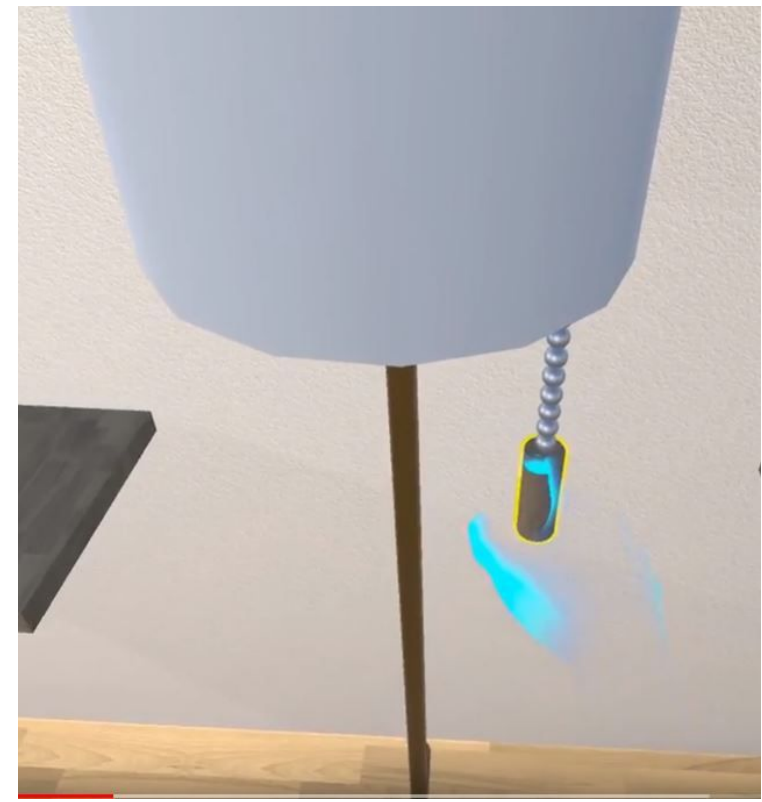

Figure 2A: Lamp

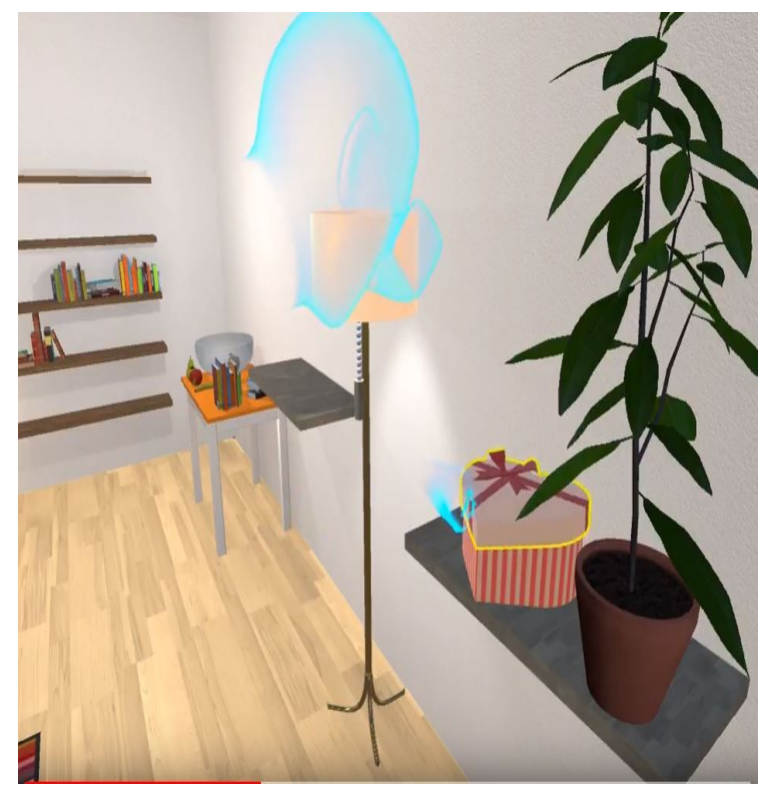

Figure 2C: Box

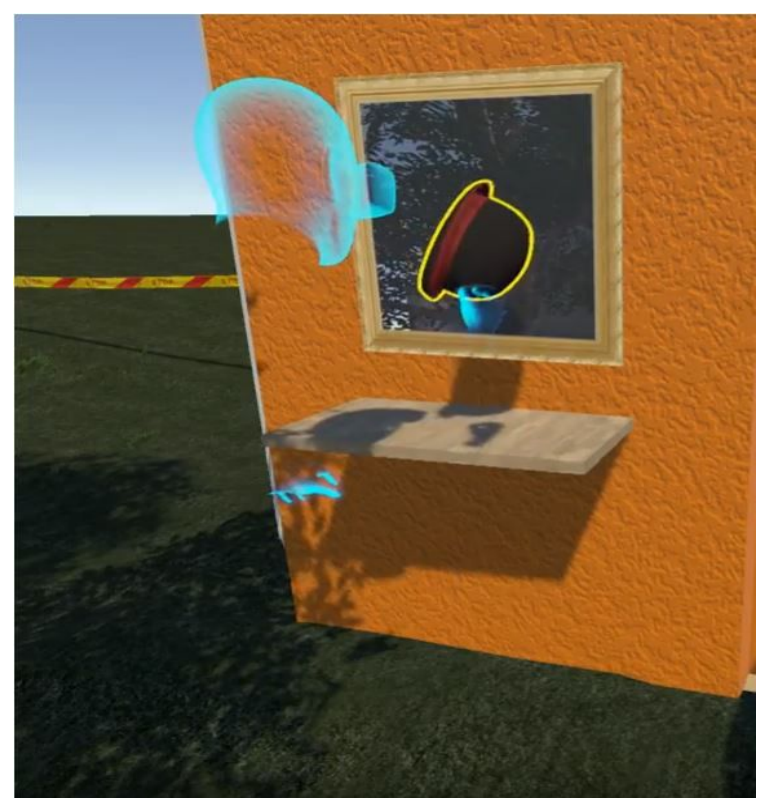

Figure 2B: Hat

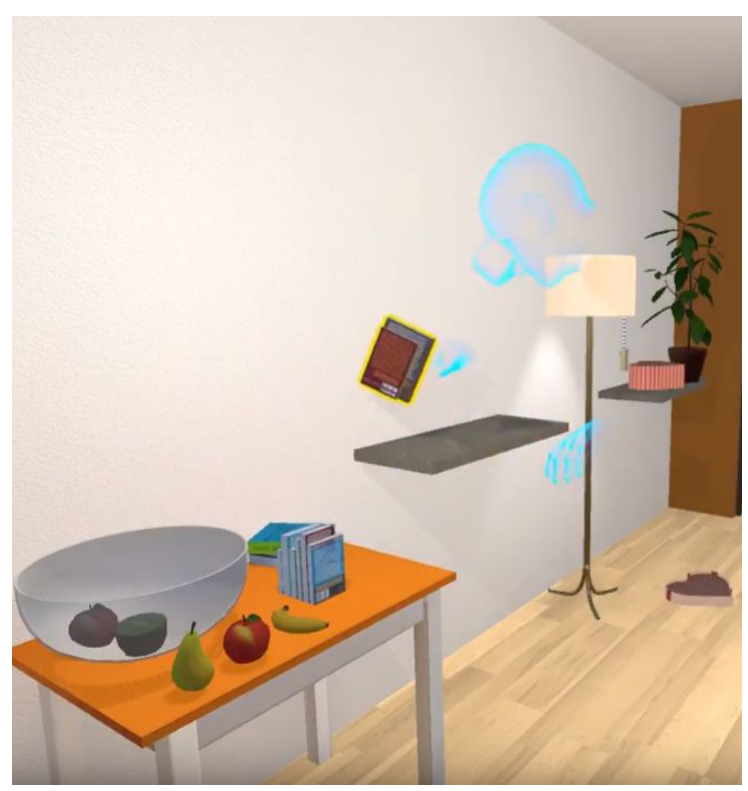

Figure 2D: Book 


\section{Appendix 1: List of all object names in English, Finnish target words, and corresponding manipulation and irrelevant movements.}

\begin{tabular}{|c|c|c|c|}
\hline $\begin{array}{l}\text { Object } \\
\text { (English) }\end{array}$ & $\begin{array}{l}\text { Target } \\
\text { Word } \\
\text { (Finnish) }\end{array}$ & $\begin{array}{l}\text { Manipulation } \\
\text { movement }\end{array}$ & Irrelevant movement \\
\hline Ball & Kera & Throw & Both hands go up to the front at the same time \\
\hline Bell & Kello & Ring & Right hand draws half a triangle, from bottom to top, inside out. \\
\hline Book & Kirja & Open & Right hand draws half a rectangle, inside out, bottom to top. \\
\hline Bottle & Pullo & Pour, drink & Right hand draws half a diamond, inside-out, bottom-up. \\
\hline Bowl & Astia & Insert into & Right hand draws half a triangle, from bottom to top, inside out. \\
\hline Broom & Luuta & $\begin{array}{l}\text { Pick up and } \\
\text { sweep }\end{array}$ & Right hand unfolds rightwards to form a straight line. \\
\hline Brush & Harja & Paint the wall & Right hand points towards the other hand and back. \\
\hline Cake & Leivos & $\begin{array}{l}\text { Pick up and } \\
\text { eat }\end{array}$ & Right hand moves up and back in a diagonal line bottom up leftwards (twice). \\
\hline Chocolate & karkki & Pick up and eat & Up and back in a diagonal line bottom up rightwards (twice). \\
\hline Door & Ovi & Open and shut & Half a circle upwards and back, counterclockwise. \\
\hline Drums & Rumpu & Beat & Half a circle downwards and back, clockwise. \\
\hline Flashlight & Sointu & Turn on & Flip right hand, extend, close, release and back. \\
\hline Flute & Pilli & $\begin{array}{l}\text { Pick up and } \\
\text { play }\end{array}$ & Right hand goes down and back up to the midline in a straight line (twice). \\
\hline Fork & Talikko & Pick up food & Right hand draws half rectangle above the left hand, and back. \\
\hline Gift & Aski & Open & Right hand down and back up to the midline in a straight line (twice). \\
\hline Glass & Lasia & $\begin{array}{l}\text { Pick up and } \\
\text { drink }\end{array}$ & Right hand draws half a circle upwards and back, counterclockwise. \\
\hline Glasses & Lasit & Wear & Right hand draws half a circle downwards and back, clockwise. \\
\hline Hammer & iskuri & Hammer a nail & Right hand draws a straight line rightwards and back. \\
\hline Hat & Myssy & Put on & $\begin{array}{l}\text { Right hand goes up with hand facing up and back with hand facing down in a } \\
\text { straight line (twice). }\end{array}$ \\
\hline
\end{tabular}




\begin{tabular}{|c|c|c|c|}
\hline Headphones & Kuulok & Put on & $\begin{array}{l}\text { Right hand draws half a circle on parallel to the ground, counterclockwise, and } \\
\text { back. }\end{array}$ \\
\hline Kettle & Pannu & Turn on & $\begin{array}{l}\text { Right hand stretches to the front, flip hand half way, move left and right, flip back, } \\
\text { move hand back. }\end{array}$ \\
\hline Key & pannu & Turn in lock & Right hand straight and up, half a circle to the left, counterclock, and back \\
\hline Lamp & Valo & Turn on to light & Right hand up and back in a diagonal line bottom up leftwards (twice). \\
\hline Leaf & lehti & Pick/pluck & Right hand goes up and back in a diagonal line top down rightwards (twice). \\
\hline Mask & Kansi & Wear & Right hand folding the arm rightwards to a right angle. \\
\hline Matchstick & Tikku & Light fire & Both arms Straighten forward and back (twice) \\
\hline Mug & Muki & Pickup, drink & Right hand does half a circle downwards and back, clockwise. \\
\hline Notebook & Vihko & $\begin{array}{l}\text { Open, sift } \\
\text { through pages }\end{array}$ & Right hand goes down and back up to the starting pose in a straight line (twice) \\
\hline Pen & Liyji & Write & Right hand draws half a triangle, from top to bottom, inside out \\
\hline Pencil & Kynä & Draw, write & Right hand draws half a diamond, inside-out, top to bottom \\
\hline Plate & Vati & Put food on it & Right hand draws half a circle downwards and back, clockwise \\
\hline Pot & Ruuku & Place on stove & Right hand draws half a rectangle, inside out, top to bottom \\
\hline Rug & Alunen & Roll & Right arm extends in front of the body and then goes down in a straight line \\
\hline Shelf & Hylly & Put books on & Right hand goes up and back in a diagonal line bottom up leftwards (twice) \\
\hline Spoon & Lusikka & Stir food & Right hand goes up and back in a diagonal line bottom up rightward (twice) \\
\hline Tap (faucet) & Hana & Turn on & Right hand goes down and back up to the midline in a straight line (twice) \\
\hline Toaster & Paahdin & $\begin{array}{l}\text { Put a slice of } \\
\text { bread in }\end{array}$ & Right hand goes down and back up to the midline in a straight line (twice) \\
\hline Trumpet & Valtti & $\begin{array}{l}\text { Pick up and } \\
\text { play }\end{array}$ & Right hand draws half a circle upwards and back, counterclockwise \\
\hline Umbrella & Turva & Open & Right hand draws half a circle downwards and back, clockwise. \\
\hline Wheel & Ratti & Roll & Right hand draws a straight angle with both hands (mirror image, inside out) \\
\hline
\end{tabular}

